\title{
Review of gene therapies for age-related macular degeneration
}

\author{
Arshad M. Khanani ${ }^{1,2}$, Mathew J. Thomas ${ }^{2}$, Aamir A. Aziz ${ }^{1,2}$, Christina Y. Weng ${ }^{3}$, Carl J. Danzig ${ }^{4,5}$, Glenn Yiu (10 ${ }^{6}$, Szilárd Kiss ${ }^{7}$, \\ Nadia K. Waheed ${ }^{8}$ and Peter K. Kaiser (iD) ${ }^{9}$
}

(C) The Author(s), under exclusive licence to The Royal College of Ophthalmologists 2021

Gene therapies aim to deliver a therapeutic payload to specified tissues with underlying protein deficiency. Since the 1990 s, gene therapies have been explored as potential treatments for chronic conditions requiring lifetime care and medical management. Ocular gene therapies target a range of ocular disorders, but retinal diseases are of particular importance due to the prevalence of retinal disease and the current treatment burden of such diseases on affected patients, as well as the challenge of properly delivering these therapies to the target tissue. The purpose of this review is to provide an update on the most current data available for five different retinal gene therapies currently undergoing clinical trials for use against age-related macular degeneration (AMD) and the development of novel delivery routes for the administration of such therapies. Research has been performed and compiled from PubMed and the select authors of this manuscript on the treatment and effectiveness of five current retinal gene therapies: Luxturna, ADVM-022, RGX-314, GT-005, and HMR59. We present the available data of current clinical trials for the treatment of neovascular and dry age-related macular degeneration with different AAV-based gene therapies. We also present current research on the progress of developing novel routes of administration for ocular gene therapies. Retinal gene therapies offer the potential for life-changing treatment for chronic conditions like age-related macular degeneration with a single administration. In doing so, gene therapies change the landscape of treatment options for these chronic conditions for both patient and provider.

Eye (2022) 36:303-311; https://doi.org/10.1038/s41433-021-01842-1

\section{INTRODUCTION}

Nearly half a century ago, Theodore Friedmann introduced the concept of gene therapy for inherited genetic disorders [1]. In theory, a gene-based therapy could afford constitutively sustained production of endogenous proteins normally absent due to underlying deficient gene efficacy. In addition, conditions that had required frequent monitoring and consistent therapeutic intervention could possibly be replaced by a single dose of a gene therapy.

The possibility of a single-dose cure for lifelong illnesses drove the development of gene therapies over the next two decades and culminated in the first FDA-approved gene therapy trial launched in 1990 to treat patients with adenosine deaminase (ADA) deficiency [2]. The success of this trial provided a milestone for gene therapies, and it became the first in a series of genetic therapy trials in the 1990s [3]. Unfortunately, subsequent trials with gene therapies were not as successful. In 1999, gene therapy for the treatment of severe combined immunodeficiency- $X 1$ ultimately resulted in the death of one patient from unforeseen oncogene activation [4]. Despite the success of previous gene therapy trials, the early ups-and-downs of gene therapy proved that there was much to be learned about the biology of viral vectors [5]. As such, subsequent research focused on the understanding of viral vectors, tissue specificity, and the genetic implications of different viral delivery methods.

\section{VIRAL VECTOR GENE THERAPY}

The concept of gene therapy is based on the introduction of a wild-type copy of a deficient or mutated gene to restore normal protein function. However, as the unexpected serious adverse reactions (SUSAR) in Hacein-Bey-Abina's clinical trial in 1999 highlighted, the challenge of introducing such a wild-type copy rests on understanding how to deliver said genes to the appropriate tissue and how to express said genes for desired therapeutic response. Viral vectors present a solution to both of these challenges given the potential for tissue specificity depending on virus type and the nature of viruses as genetically-loaded systems. The challenge then was in selecting viral vectors while taking into consideration the possibilities for host immune response and genomic integration.

Two strategies regarding gene therapy have been evaluated. Firstly, genomically-integrated vectors such as retroviral vectors could be injected with the goal being to eintroduce and transduce plasmid sequences coding for deficient protein production into the cells of affected tissue [6]. The benefit of this modality is that transduction would allow for stable gene expression in dividing and nondividing cells. This is in contrast to non-integrating viral vectors where long-lasting gene expression is less likely in highly mitotic cells lines due to loss of episome plasmid DNA [7]. The primary concerns with retroviral vectors include possible host immune response and the possibility of accidental insertional

\footnotetext{
${ }^{1}$ Sierra Eye Associates, Reno, NV, USA. ${ }^{2}$ The University of Nevada, Reno School of Medicine, Reno, NV, USA. ${ }^{3}$ Department of Ophthalmology, Baylor College of Medicine, Houston, TX, USA. ${ }^{4}$ Rand Eye Institute, Deerfield Beach, FL, USA. ${ }^{5}$ Florida Atlantic University, Charles E. Schmidt College of Medicine, Boca Raton, FL, USA. ${ }^{6}$ Department of Ophthalmology \& Vision Science, University of California, Davis, Sacramento, CA, USA. ${ }^{7}$ Department of Ophthalmology, Weill Cornell Medical College, New York-Presbyterian Hospital, New York, NY, USA. ${ }^{8}$ Department of Ophthalmology, Tufts University School of Medicine, Boston, MA, USA. ${ }^{9}$ Cole Eye Institute, Cleveland Clinic, Cleveland, OH, USA. ${ }^{\circledR}$ email: arshad. khanani@gmail.com
}

Received: 1 June 2021 Revised: 25 October 2021 Accepted: 3 November 2021

Published online: 11 January 2022 
mutagenesis. In other words, the integrating viral vectors could insert their plasmids into oncogenic or tumor-suppressing regions of DNA. Consequently, regulatory regions in the intended therapeutic plasmid could then inadvertently dysregulate cell cycle mechanisms [8].

The other modality of gene expression is to introduce a transgene through a non-integrating viral vector. The benefit over integrating vectors is that insertional mutagenesis is extremely unlikely, avoiding one of the main safety concerns of viral gene therapy. Addressing that safety concern alone makes nonintegrating viral vectors worthy of consideration.

\section{Adeno-associated viral (AAV) vectors}

In the 1990's, Kessler et al. and Xiao et al. proved that long-term expression of a transgene is possible with the in vivo administration of AAV vectors into mouse muscle $[9,10]$. These experiments laid the groundwork for exploring AAV vectors as one of the futures of gene delivery systems. Further research expanded on the solutions provided by AAV vectors as modes of therapeutic relief.

AAV vectors provided a breakthrough in genetic therapy payload delivery. First, the parent virus, adeno-associated virus, has no disease associated with it alone since AAV requires a helper virus for replication [11]. Second, the AAV genome can be edited to remove the open reading frame-encoded proteins (rep) required for viral replication and integration. If not removed, site-specific integration on chromosome 19 occurs $[12,13]$. The ability to remove rep and limit the possibilities of genome integration from the AAV plasmid offers a contrast between two different modalities in AAV vector-based gene therapy: nongenomic integration and genomic integration.

With non-genomic integration, the rep gene of AAV can be removed to limit the plasmid's capacity to replicate on its own. The obvious disadvantage of this approach is that multiple injections would be required for sustained therapeutic effect. However, sustained transgene expression can be maintained for an extended period of time in cells with a low mitotic index. On the other hand, controlled gene integration of AAV at specific, well-mapped loci on chromosome 19 would allow for long-term expressions of therapeutic plasmid. In doing so, AAV vectors and their transgene payload could convert natively deficient tissue into a transgene biofactory, negating the need for repeat injections. In other words, AAV vectors with a therapeutic plasmid construct could be used to reverse the genetic pathologies underlying a condition and restore normal function of the targeted cell type with a single dose. This is of particular importance if the mode of injection requires extensive technical skill or presents a barrier to patient comfort. More importantly, this integrated transgene modality offers the opportunity to reverse the effects of chronic illness otherwise controlled by a lifetime of medical or pharmacological intervention with a single-dose injection.

$A A V$ vectors exhibit even more flexibility with the existence of multiple serotypes. The various serotypes differ by their capsid components. Consequently, the different AAV serotypes display differential transduction efficiency, immunogenicity, and cellular tropism, and could thus be optimized to any tissue expressing underlying gene deficiency [14].

Finally, AAV vectors possess the ability to circumvent a host immune response almost entirely [15]. Despite the expectation that gene therapies should have a baseline safety requirement of minimizing a host immune or inflammatory reaction, the immune system presents a distinct challenge to viral gene therapy vectors. Unlike its parent adenovirus, AAV vectors have been shown to elicit a relatively mild immune response. This is in part because of $A A V$ 's restricted ability to infect antigen presenting cells [15]. The benefits of AAV's immunocompetence are profound. Primarily, the safety concerns around inoculation with AAV vectors are minimized if a host immune reaction can be avoided. Second to AAV's ability to avoid immune detection is that the overall vector dose required for therapeutic relief is reduced. A common workaround for eliciting a temporary immune response with other viral vectors is to increase the viral load upon delivery to overwhelm the host immune reaction. However, the increased viral load could paradoxically make the immune response more severe. For this reason, the mitigated risks provided by AAV vector therapeutics warranted further exploration in clinical trials.

\section{Retinal gene therapy}

The advances in AAV vector production, efficacy, and flexibility triggered a new wave of clinical activity in the 2000s. Researchers now had the capacity to introduce therapeutic plasmids with minimal concern for immune or inflammatory reaction with a degree of tissue specificity never seen before. With a firm understanding of AAV vectors and gene therapy biology, ocular disorders became a target for gene therapy trials. Although many disorders are often polygenic in dysfunction, ocular disorders are well-understood for having individual genes responsible for a variety of conditions [16]. This makes ocular disorders appealing since replacing a single gene both minimizes the payload delivery for AAV vectors while avoiding potential errors that can arise with large transgenes. Secondly, the potential for a single dose of gene therapy delivery is a significant improvement over the treatment burden of frequent intravitreal injections to treat retinal diseases.

These strengths of AAV permitted its use in the earliest clinical trials of ocular gene therapy. In three separate trials started in 2008, AAV2 vectors carrying a functional copy of retinal pigment epithelium (RPE)-specific $65-\mathrm{kDa}$ protein gene (RPE65) were injected subretinally into patients with RPE65-Leber congenital amaurosis. In all three trials, significant improvement was seen in improving visual dysfunction [17-19]. In doing so, the promise of gene therapy lived up to the expectations originally set by Theodore Friedmann. The results of these three trials set the groundwork leading to the FDA-approval of voretigene neparvovec-rzyl (Luxturna), the first FDA-approved ocular gene therapy.

\section{RETINAL GENE THERAPIES}

\section{Voretigene neporvovec-rzyl (Luxturna)}

Voretigene neporvovec-rzyl from Spark Therapeutics is a one-time AAV2-based subretinal gene therapy indicated in patients confirmed with biallelic RPE65 mutation-associated retinal dystrophy which manifests either as Leber congenital amaurosis 2 or rare forms of retinitis pigmentosa (RP). LCA affects 1 in $30,000-81,000$ people and is a severe and early type of inherited retinal disease-causing childhood blindness [20]. LCA is characterized by significant visual impairment starting in infancy or early childhood, searching nystagmus, a sluggish to near-absent pupillary response, and severely subnormal to non-detectable electroretinography [20, 21].

Voretigene neparvovec-rzyl works by delivering a functional copy of the gene RPE65 to retinal cells via subretinal injection. Normal RPE65 protein converts all-trans-retinol to 11-cis-retinol, allowing the formation of chromophore 11-cis-retinal during the retinoid cycle; this process is vital to the biological conversion of light photos into electrical signals transported to the brain via the optic nerve. In LCA, reduced or absent levels of RPE65 disrupts the retinoid cycle, resulting in vision impairment [22]. By transducing the RPE cells with the transgene, functional RPE65-specific kiloDalton protein is thereby produced.

The approval of voretigene neparvovec-rzyl not only made it the first-ever ocular gene therapy to become available to patients, but also represented the culmination of a large body of previous scientific work. Aside from the earliest studies described above, many other early-phase studies have been conducted including 
one Phase $1 / 2$ trial involving 12 participants who received unilateral, subretinal injections of AAV2-hRPE65v2 in the worseseeing eye in a dose-escalation study evaluating two doses, $1 \times$ $10^{10}$ and $1 \times 10^{11}$ vector genomes [18, 19, 23-27]. One patient improved visually in both the treated and untreated eyes while 3 patients experienced a visual decline. Six patients improved in dark-adapted perimetry while 3 improved in low-light navigation, and 5 patients improved in microperimetry. These effects appeared to wane beyond the first-year post-treatment. Additionally, no major systemic immune-related adverse events (AE) were noted, although 6 of 10 patients with foveal-involving injections had retinal thinning and 3 patients in the higher-dose group were observed to have intraocular inflammation.

Ultimately, the encouraging results of the Phase $1 / 2$ trial led to a Phase 3 trial concluding that voretigene neparvovec-rzyl was welltolerated overall with no product-related serious adverse effects or immune responses noted. The primary outcome evaluated the change in the performance of a study-specific multi-luminance mobility test (MLMT) from baseline at 1 year, which was met as the treated group made statistically significant improvements in their end score. Secondary endpoints evaluated full-field stimulus thresholds and Goldmann visual field testing, with significant improvements for both measures in the treated group over the control group [28]. The most common ocular adverse effects were surgery-related and included elevated intraocular pressure (20\%), cataracts $(15 \%)$, ocular inflammation $(10 \%)$, and retinal tears $(10 \%)$.

These overall favorable results observed in this Phase 3 trial led to the approval of voretigene neparvovec-rzyl for the treatment of biallelic RPE65 deficient-associated retinal disease by the United States Food \& Drug Administration in December 2017. Voretigene neparvovec-rzyl is currently being administered at ten treatment centers in the United States.

\section{ADVM-022}

ADVM-022 by Adverum Biotechnologies is currently in a Phase 1 open-label clinical trial (OPTIC) as an in-office intravitreal gene therapy to treat neovascular age-related macular degeneration
(nAMD), the most common cause of vision impairment in individuals 50 years of age and older, and a Phase 2 trial (INFINITY) for the treatment of diabetic macular edema [29].

Pathologically, vascular endothelial growth factor (VEGF) promotes the development of choroidal neovascularization and the subsequent progression of nAMD. As such, anti-VEGF therapies have been the primary focus of pharmacological intervention for individuals with nAMD. Aflibercept is an antiVEGF protein which is commonly used in the treatment of retinal diseases and has been FDA-approved since 2011. ADVM-022 aims to treat $n A M D$ through a single intravitreal injection utilizing the AAV.7m8 capsid to deliver a codon-optimized CDNA expressing an aflibercept-like protein (Fig. 1). With ADVM-022, the need for repeated intravitreal injections every $4-8$ weeks can be mitigated with a resulting decrease in treatment burden [30].

Nonhuman primate studies with ADVM-022 have found that a single intravitreal injection of ADVM-022 resulted in sustained expression of aflibercept for 30 months [30]. Moreover, the aflibercept was able to inhibit choroidal neovascularization in a nonhuman primate model of nAMD, even when injected 13months prior to lasering [31]. These findings led to the initiation of the OPTIC trial, which is the first in-human trial with ADVM-022 for patients with nAMD. The 2-year ongoing OPTIC Phase I trial has enrolled 4 cohorts of previously-treated patients with neovascular AMD: cohorts 1 (6 patients) and 4 (9 patients) received the high dose $\left(6 \times 10^{11} \mathrm{vg} / \mathrm{eye}\right)$, and cohorts 2 (6 patients) and 3 ( 9 patients) received the low dose $\left(2 \times 10^{11} \mathrm{vg} / \mathrm{eye}\right)$. These patients have been heavily pre-treated prior to enrolling in OPTIC with an average of 9 injections in the previous year in cohorts 1,2, and 3, with 7 injections in cohort 4 . The primary outcome evaluates the safety and tolerability of one injection of ADVM-022, while the second objectives assess the best-corrected visual acuity (BCVA), anatomic outcomes of spectral-domain optical coherence tomography, and the need for anti-VEGF supplemental therapy. Steroid prophylaxis of ocular inflammation was used in all 4 cohorts, but with different regimens per cohort. A 13-day course of oral steroid with tapered topical eyedrops were used in cohorts 1 and 2 while cohorts 3 and

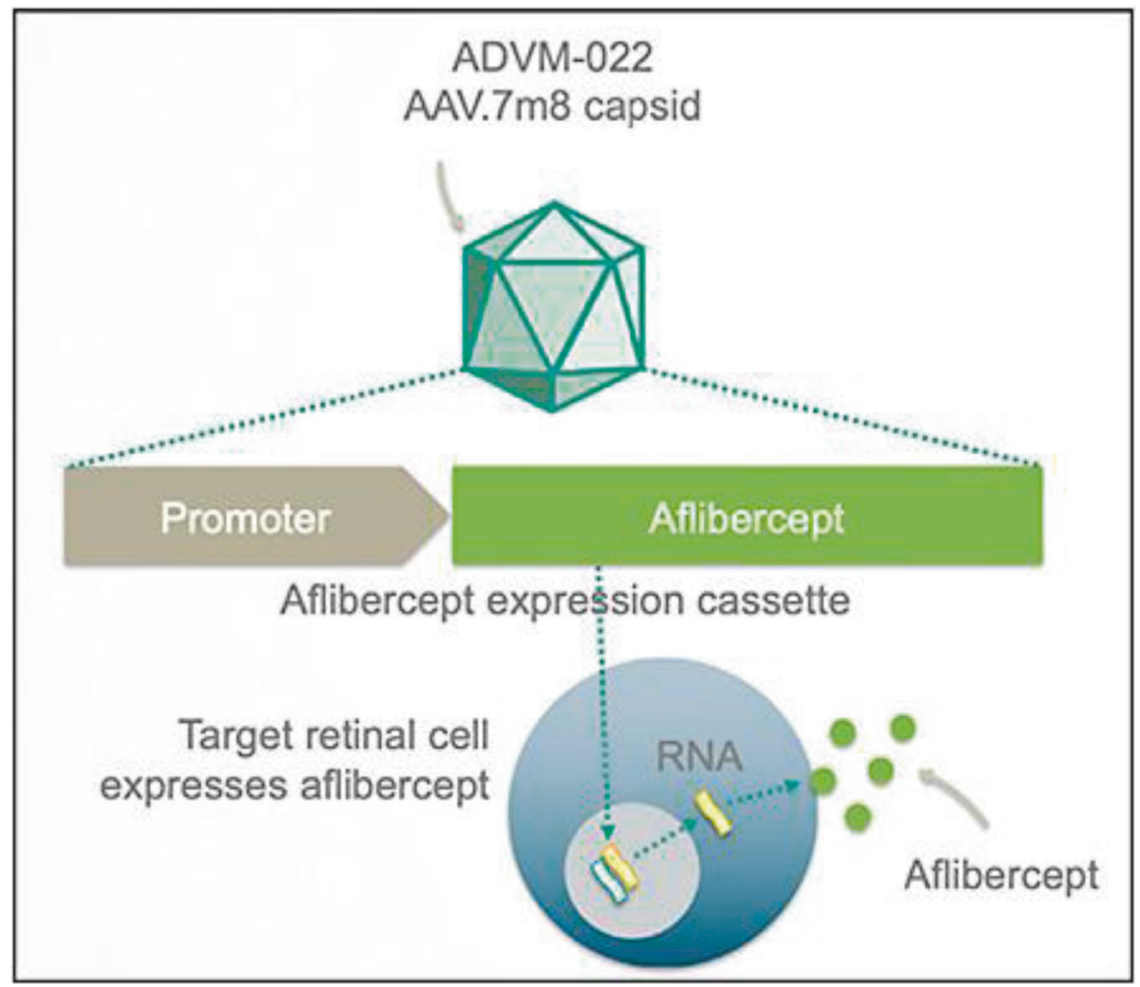

Fig. 1 ADVM-022 mechanism of action. 
4 were treated with a 6-week course of topical steroid eyedrops. The cohorts have similar demographics and anatomical baselines with cohort 3 having a higher average central subfield thickness (CST) at baseline.

Following a single intravitreal injection of ADVM-022, the highdose cohorts demonstrated a $96 \%$ reduction in the mean annualized frequency of anti-VEGF injections and the low-dose cohorts demonstrated an $85 \%$ reduction in treatments [32].

The BCVA and CST in cohort 1 remained stable without supplemental treatment at a median 104-week follow-up [32, 33]. Subjects maintained -1.3 letters and $-8.7 \mu \mathrm{m}$ reduction in CST during this time period. Cohort 2 maintained BCVA ( -1.5 letters in all patients and -1.0 letters in supplemental-free patients) and CST $(-28.2 \mu \mathrm{m}$ in all patients and $-30.3 \mu \mathrm{m}$ in supplemental-free patients) over a median 84-week follow-up. In cohort 3, the BVCA (+1.4 letters in all patients and +4.3 letters in supplemental-free patients) was maintained and the CST $(-134.4 \mu \mathrm{m}$ in all patients and $-181.7 \mu \mathrm{m}$ in supplemental-free patients) improved through a median 56-week follow-up. In cohort 4, the BVCA ( -0.2 letters in all patients and -0.4 letters in supplemental-free patients) was maintained and the CST $(-77.1 \mu \mathrm{m}$ in all patients and $-77.3 \mu \mathrm{m}$ in supplemental-free patients) improved through a median 36-week follow-up.

No ADVM-022-related non-ocular AEs have been reported at this time. Inflammation has been the main AE in this trial, with ocular inflammation predominately affecting the anterior segment noted especially in the higher-dose cohorts. No posterior inflammation, vasculitis, or endophthalmitis has been reported. A decreasing trend in inflammation has been seen over time but multiple patients have required long-term topical drops, particularly in the high-dose group. The lower dose has been associated with fewer cases of inflammation. All treatment-related ocular AEs were mild $(80 \%)$ to moderate $(20 \%)$. An AE of special interest (AESI) was documented in the high-dose Cohort 1 of moderate recurrent uveitis unrelated to ADVM-022 treatment. This patient was positively responsive to steroid eyedrops. Two patients had mild AEs of IOP elevation that resolved with Combigan eyedrops [34].

Importantly, in those patients who consented for aqueous taps in the OPTIC trial, there was sustained long-term aflibercept protein expression, out to 104 weeks in one patient. With the exception of one patient who required rescue injections, all aflibercept protein levels expressed as a result of ADVM-022 gene therapy were found to be in therapeutic range when compared to the commercially available Eylea (aflibercept recombinant protein, Regeneron Pharmaceuticals) injection [30, 32, 35].

As this trial is ongoing and a long-term extension is planned, we will continue to learn more about safety and efficacy of ADVM022. These results will guide the design of future studies to evaluate ADVM-022 as a potential option for the treatment of nAMD.

Adverum Biotechnologies conducted a second trial, INFINITY, for the use of ADVM-022 for diabetic macular edema (DME). In late April of 2021, Adverum unmasked the trial following a SUSAR of hypotony in the treated eye of a DME patient in the high-dose cohort of the trial 16-36 weeks after administration of ADVM-022. Close monitoring and aggressive treatment led to no improvement in the patient's condition. Similar SAEs have not been observed in the low-dose cohorts. Adverum has discontinued development and investigation of ADVM-022 for DME at this time [36].

\section{RGX-314}

RGX-314 (REGENXBIO Inc.) uses an AAV8 associated gene therapy for the treatment of nAMD. RGX-314 expresses a monoclonal antibody fragment similar to ranibizumab. As an established antiVEGF therapy, ranibizumab is a humanized monoclonal antibody fragment that binds to human VEGF-A to suppress choroidal neovascularization [37]. RGX-314 uses subretinal or suprachoroidal delivery and offers the potential for stable anti-VEGF antibody production while reducing the burden of multiple intravitreal injections (Fig. 2).

42 patients with severe nAMD have been enrolled in the ongoing Phase I/lla trial of RGX-314 looking at safety and efficacy of RGX-314 delivered subretinally via transvitreal approach. The therapy has been generally well-tolerated with no reports of abnormal immune response, drug-related ocular inflammation, or post-surgical inflammation made thus far [38]. 20 SAEs have been reported in 13 patients with one possibly drug-related SAE of significant decrease in vision in the high-dose Cohort $5.87 \%$ of the most common nonserious ocular AEs were assessed as mild with the common AEs being postoperative conjunctival hemorrhage in $67 \%$ of patients, post-operative inflammation in $36 \%$ of patients, and eye irritation eye pain, and postoperative visual acuity reduction in $17 \%$ of patients each [37]. There

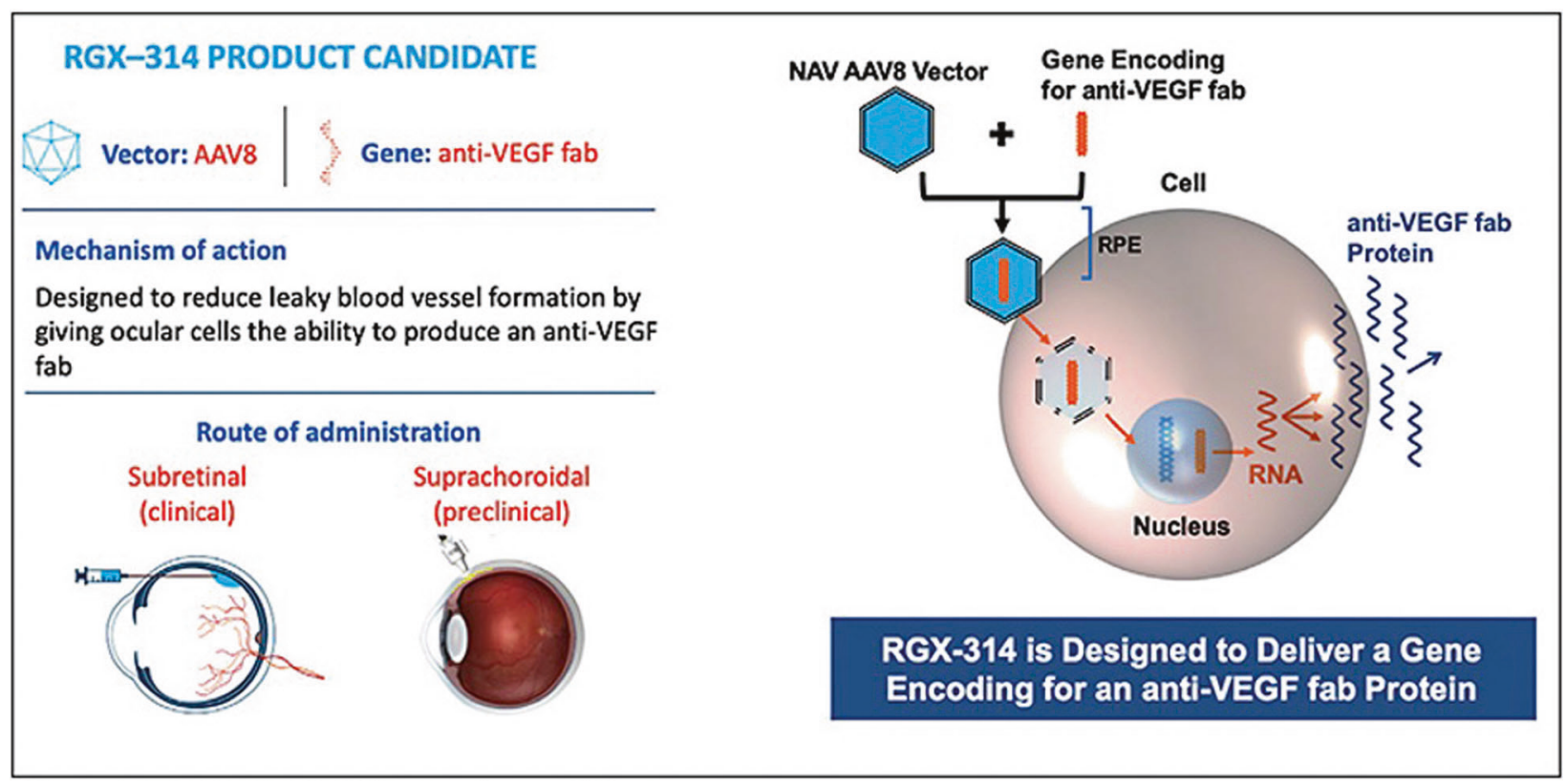

Fig. 2 [37] RGX-314 mechanism of action. 
has also been no drug-related ocular inflammation, abnormal postsurgical inflammation, or clinically determined immune responses observed to date [38].

Retinal pigmentary changes at the site of the subretinal bleb have been noticed in $67 \%$ of the patients. Thus far, the efficacy data from the trial has been promising. Patients in cohorts 3, 4, and 5 have included 6,12 , and12 patients respectively at the doses of $6 \times 10^{10}$ GC/eye $1.6 \times 10^{11} \mathrm{GC} /$ eye and $2.5 \times 10^{11} \mathrm{GC} /$ eye respectively. 2-year data from cohort 3 has shown BCVA gains of +14 letters. In cohort 4 and 5 , at 1.5 years, mean BCVA in these two cohorts has changed +1 letters and -1 letters from baseline respectively. Central retinal thickness has changed $+2 \mu \mathrm{m}$ in cohort 3 and -46 and $-93 \mu \mathrm{m}$ in cohorts 4 and 5 respectively. Patients in cohort 3 have benefited from a decrease in mean change in annualized injection of $66.7 \%$ at 3 years while cohort 4 and cohort 5 patients have experienced a $58.3 \%$ and $81.2 \%$ reduction, respectively, at 1.5 years.

The study is ongoing and patients will be followed in the extension trial for a total of 5 years from enrollment. We will continue to learn about efficacy and safety of RGX-314 through other studies and an expanding clinical program. The pivotal ATMOSPHERE trial is evaluating subretinal injections of RGX-314 for nAMD patients. The Phase 2 trials AAVIATE and ALTITUDE for neovascular AMD and diabetic retinopathy respectively, will assess RGX-314 administered via the suprachoroidal route.

\section{GT-005}

Gyroscope's Therapeutics' FOCUS trial utilizing GT-005 is a gene therapy designed to target patients with dry AMD. Dry AMD accounts for $85-90 \%$ of all AMD cases [39]. Unlike nAMD, there are currently no effective therapies available for dry AMD. Advanced dry AMD, also called geographic atrophy (GA), is characterized by irreversible degeneration of RPE cells and their overlying retinal photoreceptors, leading to permanent vision loss.

Though there are no established treatments for dry AMD, one proposed pathological mechanism involves dysregulation of the complement system. It is believed that an overactive alternative pathway of the complement system, an arm of the innate and adaptive immune systems, is partially responsible for the development of dry AMD. This hypothesis is supported by Gene-byEnvironment Wide Association Studies (GEWAS) and the accumulation of complement proteins in drusen which are insoluble extracellular aggregates in the retina characteristic of AMD [40].

GT-005's therapeutic concept is the AAV2 vector delivering a plasmid construct expressing normal Complement Factor I (CFI) protein, a natural inhibitor of the complement system [41]. GT-005 is designed to enable cellular transduction and induce secretion of CFI. It has the potential to allow for constitutive expression of CFI after a single administration and to avoid the sawtooth dynamics of repeated intravitreal injections. CFI is a natural regulator and functions to keep the complement system in balance. Given this critical role, CFI is well suited for gene therapy. GT-005 is currently undergoing evaluation for safety and efficacy in multiple Phase 1 (FOCUS) and Phase 2 (HORIZON and EXPLOREe) clinical trials.

At this time, no GT-005- related SAEs have been observed. 1 possible GT-005-related AE of choroidal neovascularization of moderate severity has been observed and treated with anti-VEGF therapy. 12 surgery-related AEs have been observed with $75 \%$ categorized as mild and $25 \%$ categorized as moderate. Two ocular AEs have been observed with increased IOP. One case of increased IOP resolved with drops. The second case self-resolved. No signs of GT-005-related inflammation or immune response have been observed to date [42].

The study is ongoing and continues to be monitored closely to assess the longer-term safety and efficacy of GT-005.

\section{HMR59}

HMR59 by Hemera Biosciences is an AAV2 vector-based gene therapy expressing sCD59 administered intravitreally 7 days after a single intravitreal injection of anti-VEGF treatment. CD59 is found on the surface of RPE cells and is believed to inhibit the formation of the MAC complex during late-stage complement system activation. CD59 prevents the formation of the MAC complex by inhibiting the recruitment of $\mathrm{C} 9$ to the $\mathrm{C} 5 \mathrm{~b}-\mathrm{C} 8$ complex [43]. By targeting the MAC complex specifically, the upstream complement cascade remains intact. Because an overactivation of the complement system is proposed to be one of the pathological pathways leading to nAMD, HMR59 aims to upregulate CD59 expression on RPE to protect against the complement cascade suspected in causing macular neovascularization.

HMR59 is currently under review in two separate Phase 1 clinical trials. Trial HMR-1002 (NCT03585556) is underway to assess the efficacy and safety of two doses of the HMR59 for treatment against nAMD. 22 patients will be injected with a low-dose treatment of $3.56 \times 10^{11} \mathrm{vg} / \mathrm{eye}$, and 3 patients will be injected with a high-dose treatment of $1.071 \times 10^{12} \mathrm{vg} /$ eye. Anti-VEGF treatments will be injected at Day 0 to treat per standard of care. The study is ongoing and will be evaluated for long-term follow-up safety and efficacy [44].

HMR59 is also being evaluated for use against dry AMD in the HMR-1001 (NCT03144999) trial. 17 patients will have been injected with either a low, mid, or high dose of HMR59 in the affected eye [45]. Thus far, HMR59 has generally been well tolerated. 3 of 17 subjects developed mild vitritis that resolved with topical steroids, A 23\% reduction in GA has been observed at the highest dose, and no treatment eyes have converted to nAMD during the 18-month follow-up. HMR-1001 is also ongoing and will be evaluated for long-term follow-up safety and efficacy [46].

\section{Routes of delivery}

Due to the complex nature of ocular anatomy, a number of options exist for ocular injections. The most common routes of ocular delivery at this time are intravitreal and subretinal injections, although suprachoroidal delivery is emerging as a novel route as well. Each offers different advantages and disadvantages that need to be explored for successful delivery of genetic therapies and subsequent treatment of ocular disease.

\section{Intravitreal injections}

Intravitreal injections have been the most widely studied and explored options for intraocular delivery, due to the ease of accessing the vitreous cavity using a needle, which can be performed in an outpatient clinical setting. The vitreous cavity allows high drug concentrations to be achieved, as with intraocular steroids and antiVEGF therapeutic agents, and can exert a broad area of therapeutic effect across the entire globe. However, intravitreal injections are susceptible to endophthalmitis, retinal detachment, and hemorrhage [47]. The injectate may interact with anterior segment structures and could cause cataracts or ocular hypertension, as with intravitreal steroids. Because the major route of egress is through the trabecular meshwork, the drug or viral particles can exit into the systemic circulation, potentially triggering host immune responses. Finally, intravitreal injections of most AAV subtypes do not readily penetrate into the neurosensory retina, due to the presence of the internal limiting membrane (ILM) which acts as a barrier to viral particles. Novel AAV subtypes that can overcome this barrier are under investigation [30].

\section{Subretinal injections}

Subretinal injections can overcome many of the limitations of intravitreal injections. Subretinal injections enable delivery of the therapeutic agent into direct contact with photoreceptors and RPE cells, bypassing the ILM barrier. This route is an efficient way to deliver interventions to focal regions of the retina (e.g. the macula) by localizing the material in the confined space of the subretinal bleb. Furthermore, subretinal injections are less likely to elicit an immune response due to their enclosure within an immune privileged location without violating the outer blood-retina-barrier. For subretinal 
injection, two routes of delivery have been utilized: (1) A transscleral route passing through the choroid without penetrating the retina and without the need for vitrectomy; (2) A transvitreal route passing through the pars plana, vitreous, and retina into the subretinal space without penetrating the choroid [47]. In humans, the most common is via the transvitreal approach, but it does require invasive vitrectomy surgery, which may be prone to complications such as hemorrhage, cataract, endophthalmitis, and retinal detachment. Transscleral routes could be achieved using a microneedle, although this cannot be easily reproduced in a reliable fashion [48]. A novel mode of delivery has been FDA approved and it involves the passage of a thin cannula through an anterior sclerotomy into the suprachoroidal space, followed by deployment of a needle through the choroid to enter the subretinal space [49].

\section{Suprachoroidal injections}

The suprachoroid is a novel route for intraocular delivery that was initially used for delivering intraocular steroids to treat the posterior retina while minimizing exposure of anterior segment structures [5052]. Due to the microscopic nature of this potential space located between the scleral wall and choroidal vasculature, microneedles have been developed to specifically access this space [53]. AAV8 delivered using suprachoroidal microneedles enabled successful transduction of multiple retinal cell types in a broad region, without the need of invasive vitreoretinal surgery [48, 54]. Because the suprachoroidal space is adjacent to the high blood flow of the choroid, injecting formulations with larger particle size such as a steroid emulsion, viral particles, or nanoparticles are necessary to avoid rapid egress from the suprachoroidal space [55]. Also, because the suprachoroidal space is outside the outer blood-retina-barrier formed by the RPE layer, there is a potential risk for host immune responses to the viral particle or transgene [56].

\section{Gene therapy-associated inflammation}

The use of viral vectors for gene delivery may result in both innate and adaptive host immune responses to either the viral vectors or the transgene. For example, humoral responses from pre-existing neutralizing antibodies (NAbs) to prior exposure to wild-type AAV that resemble the capsid of the AAV vector may limit successful gene transfer by inhibiting transduction. Also, cell-mediated responses from $T$ cells can destroy successfully transduced cells. Thus, both humoral and cellular immune responses contribute to reducing the effectiveness of gene therapies by eliminating vectors and transduced cells [57].

Viral-mediated gene therapies can trigger ocular inflammation in the form of anterior uveitis or iritis, as well as vitritis or chorioretinitis. The degree and type of inflammation is generally dictated by the type of viral vector, promoter, and transgene, but more importantly is heavily affected by dose and route of delivery. For example, adenovirus triggers robust intraocular inflammation compared to $A A V$, although some AAV serotypes are associated with pre-existing immunity [58]. NAbs against AAV1 and 2 have the highest prevalence with rates between 30 and $70 \%$, while AAV5, 7, 8, and 9 are lower at 15-30\% [59]. Whether these pre-existing NAbs negatively impacts gene transduction remains a topic of controversy, however. The type of transgene may also impact host immune responses, since foreign proteins such as GFP which is commonly used in preclinical studies as a marker for testing transduction efficiency, are cytotoxic [60]. Also, the host immune system in patients with inborn genetic mutations may recognize the wild-type transgene product as foreign, as in AAVmediated gene therapy for Duchenne muscular dystrophy [61]. Interestingly, ubiquitous promoters such as cytomegalovirus and chicken beta actin may cause more retinal toxicity than photoreceptor-specific promoters such as rhodopsin kinase [62].

Beside the type of viral vector, transgene, or promoter, perhaps the more important factor that determines host immunity is the viral dose and route of delivery. Ocular inflammation tends to occur at lower doses with intravitreal injections. Patients that received the highest doses of intravitreal AAV2-sFlt01 for neovascular AMD $\left(2 \times 10^{8}-2 \times 10^{11} \mathrm{vg}\right)$, or intravitreal AAV2-RS1 for $X$-linked retinoschisis $\left(1 \times 10^{10}-1 \times 10^{11} \mathrm{vg}\right)$ developed antiAAV2 antibodies and intraocular inflammation that responded to topical or oral steroids [63]. By contrast, subretinal injections generally exhibit less inflammatory responses at similar doses. Long-term safety data from Phase 3 studies for voretigene neparvovec-rzyl showed no significant inflammatory responses up to 4 years after treatment [64]. In patients who received subretinal AAV2-REP1 for choroideremia, only 1 in 14 who received the higher $1 \times 10^{11} \mathrm{vg}$ dose showed retinal inflammation that was later attributed to vector reflux into the vitreous cavity after subretinal injection [65]. The mechanism for this distinction based on delivery route may result from differences in vector egress and systemic biodistribution. In nonhuman primate studies, intravitreal AAV showed 464-fold higher outflow into systemic circulation than subretinal injections, with higher levels detected in the spleen and draining lymph nodes [66]. Early studies using suprachoroidal AAV8 showed less NAb responses to the vector, but more $\mathrm{T}$-cell response to the transgene, presumably due to lower outflow of the AAV from the suprachoroidal space than vitreous cavity, but greater GFP expression in the sclera which is outside the blood-retinal barrier, and therefore does not benefit from immune privilege [56].

\section{Gene therapy vs gene editing}

In contrast to standard gene augmentation therapy, where a viral or non-viral delivery vector is used to introduce a therapeutic transgene to replace a defective mutant gene, or to generate an inhibitory protein to suppress an aberrant or pathogenic protein, gene editing involves manipulation of the target gene at the DNA or genomic level. The most common gene editing system to date uses clustered regularly interspaced short palindromic repeats (CRISPR) endonucleases such as Cas9, which can cut the DNA at a precise, targeted location, to either ablate or repair a destructive mutation [67]. When the DNA is cut, the cell attempts to repair it through mechanisms that are error-prone and causes random insertions or deletions to cause a frameshift mutation and effectively disable the gene. If a therapeutic, normal copy of a gene segment is also provided, a process known as homologous recombination may occur to replace the mutation with the correct sequence, although this process is not very efficient. Newer, more precise strategies that do not involve double-strand breaks or donor DNA templates include base editing, where a single nucleotide is modified, and prime editing, which involves "nicking" single DNA strands, although these techniques are still under investigation [68-70]. Therefore, while gene augmentation therapies are optimal for inherited conditions with recessive mutations or haploinsufficiency, gene editing may be better suited to ablate or disrupt dominant mutations.

Early success using CRISPR-based gene editing was achieved in animal models of inherited retinal disorders, as by inactivating the rhodopsin mutations in RP mouse models, or by disrupting the splice mutation in CEP290 gene in LCA type 10 mice [71-74]. Due to the heterogeneity of different mutations, another unique application couples CRISPR-Cas9 disruption of the endogenous rhodopsin gene with exogenous expression of wild-type rhodopsin in a process known as an "ablate-and-replace" approach [71]. CRISPR-Cas9 has also been used to suppress VEGF secretion from human RPE cells, and mouse models of CNV [75, 76]. Early phase $1 /$ II studies are under way to evaluate CRISPR-based strategies for treatment of LCA10. Although gene editing, like gene therapy, has the potential to radically improve human health, the concept of directly manipulating the human genome has been subject to moral and ethical debates. Despite the differences in conceptual basis, mechanisms of action, and advantages and disadvantages, gene therapy and gene editing are undoubtedly part of the future, if not the future, of therapeutic intervention. 

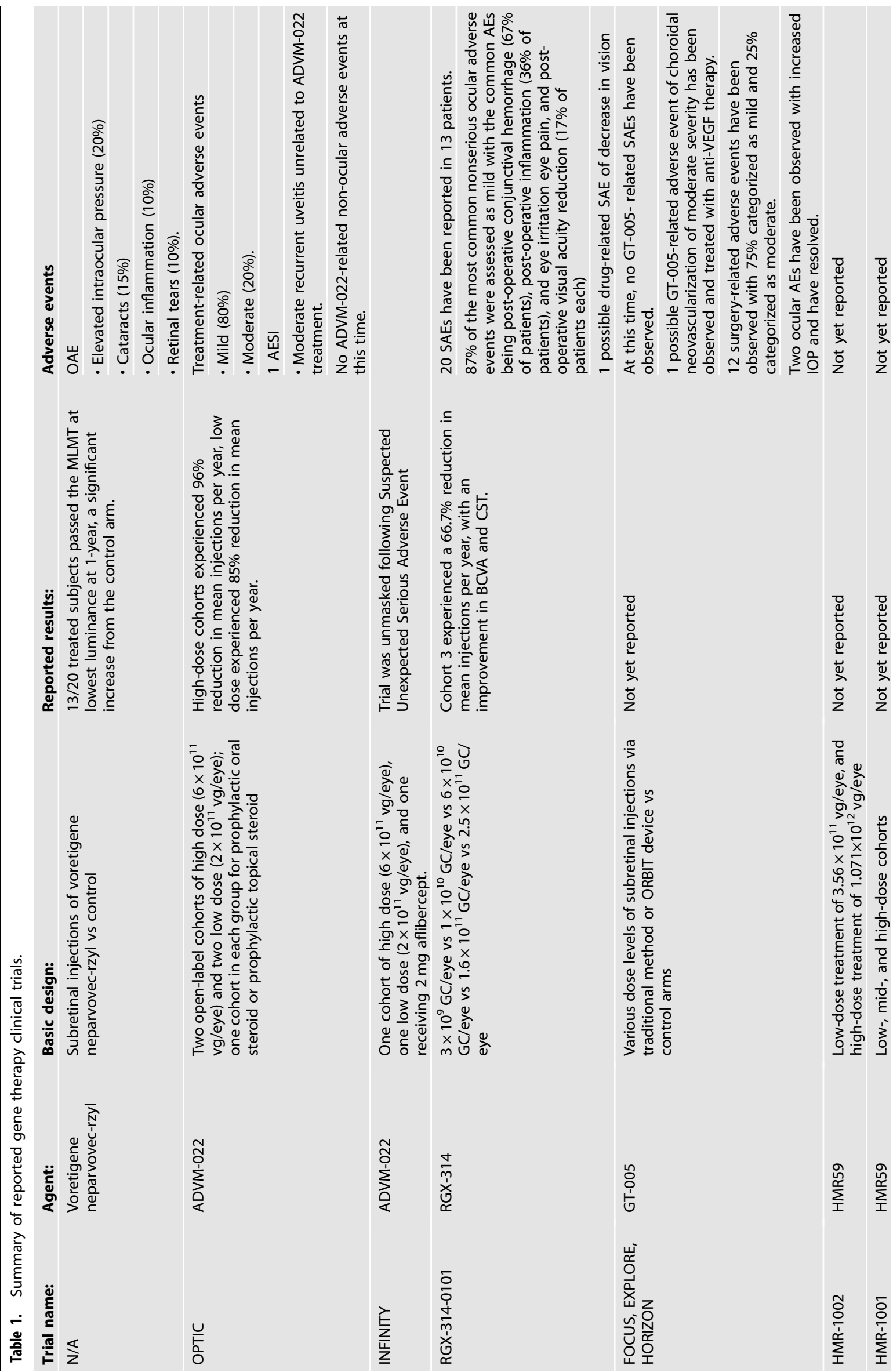


\section{DISCUSSION}

Gene therapy offers a degree of innovation and intervention unlike anything seen before. In just five decades, gene therapy evolved from a simple concept on paper to the scientific subject of over 1400 clinical trials across the globe.

Viral vector gene therapy appears to be an innovative treatment option in the management of retinal diseases like wet and dry AMD. In addressing the treatment burden currently in place for such conditions, gene therapy sets itself up to be at the forefront of retinal therapies in the coming decades. Namely, as discussed here, the results of the ADVM-022, RGX-314, GT-005, and HMR59 could revolutionize treatment plans for wet and dry AMD (Table 1). Progress has been made to optimize dosage of these drugs, routes of administration, management post-injection, and long-term benefits.

Furthermore, the coming years will show improvements in reducing one of the greatest limiting factors of gene therapy as a therapy modality: cost. Optimizing vector production, tissue specificity, and transgene expression will be of utmost importance in limiting excessive costs with gene therapy. That said, the cost of gene therapy, and especially retinal gene therapies, are for a onetime administration [77].

The overall results of the trials discussed and the advancement of the fields of gene therapy and gene editing make retinal gene therapies a dynamic and ever-evolving field with plenty to look forward to for both the betterment of our patients and the advancement of clinical potential.

\section{REFERENCES}

1. Friedmann T, Roblin R. Gene therapy for human genetic disease? Science. 1972;175:949-55.

2. Blaese RM, Culver KW, Miller AD, Carter CS, Fleisher T, Clerici M, et al. T lymphocyte-directed gene therapy for ADA- SCID: initial trial results after 4 years. Science. 1995;270:475-80.

3. Tamura R, Toda M. Historic overview of genetic engineering technologies for human gene therapy. Neurol Med Chir. 2020;60:483-91.

4. Hacein-Bey-Abina S, von Kalle C, Schmidt M, Le Deist F, Wulffraat N, Mclntyre E, et al. A serious adverse event after successful gene therapy for X-linked severe combined immunodeficiency. N. Engl J Med. 2003;348:255-6.

5. Dunbar CE, High KA, Joung JK, Kohn DB, Ozawa K, Sadelain, M. Gene therapy comes of age. Science. 2018;359.

6. Scheller EL, Krebsbach PH. Gene therapy: design and prospects for craniofacial regeneration. J Dent Res. 2009;88:585-96.

7. Athanasopoulos T, Munye MM, Yáñez-Muñoz RJ. Nonintegrating gene therapy vectors. Hematol Oncol Clin North Am. 2017;31:753-70.

8. Nienhuis AW, Dunbar CE, Sorrentino BP. Genotoxicity of retroviral integration in hematopoietic cells. Mol Ther. 2006;13:1031-49.

9. Kessler PD, Podsakoff GM, Chen X, McQuiston SA, Colosi PC, Matelis LA, et al. Gene delivery to skeletal muscle results in sustained expression and systemic delivery of a therapeutic protein. Proc Natl Acad Sci USA. 1996;93:14082-7.

10. Xiao X, Li J, Samulski RJ. Efficient long-term gene transfer into muscle tissue of immunocompetent mice by adeno-associated virus vector. J Virol. 1996;70:8098-108.

11. Naso MF, Tomkowicz B, Perry WL 3rd, Strohl WR. Adeno-associated virus (AAV) as a vector for gene therapy. BioDrugs. 2017;31:317-34.

12. Kotin RM, Siniscalco M, Samulski RJ, Zhu XD, Hunter L, Laughlin CA, et al. Sitespecific integration by adeno-associated virus. Proc Natl Acad Sci USA. 1990;87:2211-5.

13. Carter B, Burstein $H$, Peluso R. Adeno-associated virus and AAV vectors for gene delivery. Gene Therapy. CRC Press; Boca Raton, FL; 2003, pp. 71-101.

14. Pillay S, Zou W, Cheng F, Puschnik AS, Meyer NL, Ganaie SS, et al. Adenoassociated virus (AAV) serotypes have distinctive interactions with domains of the cellular AAV receptor. J Virol. 2017;91. https://doi.org/10.1128/JVI.00391-17.

15. Bessis N, GarciaCozar FJ, Boissier M-C. Immune responses to gene therapy vectors: influence on vector function and effector mechanisms. Gene Ther. 2004;11: S10-7.

16. Rodrigues GA, Shalaev E, Karami TK, Cunningham J, Slater NKH, Rivers HM. Pharmaceutical development of AAV-based gene therapy products for the eye. Pharm Res. 2018;36:29.

17. Bainbridge JWB, Smith AJ, Barker SS, Robbie S, Henderson R, Balaggan $K$, et al. Effect of gene therapy on visual function in Leber's congenital amaurosis. N. Engl J Med. 2008:358:2231-9.
18. Maguire AM, Simonelli F, Pierce EA, Pugh EN Jr, Mingozzi F, Bennicelli J, et al. Safety and efficacy of gene transfer for Leber's congenital amaurosis. N. Engl J Med. 2008;358:2240-8.

19. Hauswirth WW, Aleman TS, Kaushal S, Cideciyan AV, Schwartz SB, Wang L, et al. Treatment of Leber congenital amaurosis due to RPE65Mutations by ocular subretinal injection of adeno-associated virus gene vector: Short-term results of a phase I trial. Hum Gene Ther. 2008;19:979-90.

20. Chacon-Camacho OF, Zenteno JC. Review and update on the molecular basis of Leber congenital amaurosis. World J Clin Cases. 2015;3:112-24.

21. Pennesi ME, Stover NB, Stone EM, Chiang P-W, Weleber RG. Residual electroretinograms in young Leber congenital amaurosis patients with mutations of AIPL1. Investig Ophthalmol Vis Sci. 2011;52:8166-73.

22. Gao J, Hussain RM, Weng CY. Voretigene neparvovec in retinal diseases: A review of the current clinical evidence. Clin Ophthalmol. 2020;14:3855-69.

23. Jacobson SG, Cideciyan AV, Ratnakaram R, Heon E, Schwartz SB, Roman AJ, et al. Gene therapy for leber congenital amaurosis caused by RPE65 mutations: safety and efficacy in 15 children and adults followed up to 3 years: Safety and efficacy in 15 children and adults followed up to 3 years. Arch Ophthalmol. 2012;130:9-24.

24. Weleber RG, Pennesi ME, Wilson DJ, Kaushal S, Erker LR, Jensen L, McBride MT, et al. Results at 2 years after gene therapy for RPE65-deficient Leber congenital amaurosis and severe early-childhood-onset retinal dystrophy. Ophthalmology. 2016;123:1606-20.

25. Chung DC, McCague S, Yu Z-F, Thill S, DiStefano-Pappas J, Bennett J, Cross D, et al. Novel mobility test to assess functional vision in patients with inherited retinal dystrophies. Clin Exp Ophthalmol. 2018:46:247-59.

26. Bennett J, Wellman J, Marshall KA, McCague S, Ashtari M, DiStefano-Pappas J, et al. Safety and durability of effect of contralateral-eye administration of AAV2 gene therapy in patients with childhood-onset blindness caused by RPE65 mutations: a follow-on phase 1 trial. Lancet. 2016;388:661-72.

27. Bainbridge JWB, Mehat MS, Sundaram V, Robbie SJ, Barker SE, Ripamonti C, et al. Long-term effect of gene therapy on Leber's congenital amaurosis. N. Engl J Med. 2015;372:1887-97.

28. Russell S, Bennett J, Wellman JA, Chung DC, Yu Z-F, Tillman A, et al. Efficacy and safety of voretigene neparvovec (AAV2-hRPE65V2) in patients with RPE65mediated inherited retinal dystrophy: a randomised, controlled, open-label, phase 3 trial. Lancet. 2017;390:849-60.

29. Grishanin R, Vuillemenot B, Sharma P, Keravala A, Greengard J, Gelfman C, et al. Preclinical evaluation of ADVM-022, a novel gene therapy approach to treating wet age-related macular degeneration. Mol Ther. 2019;27:118-29.

30. Kiss S, Oresic Bender K, Grishanin RN, Hanna KM, Nieves JD, Sharma P, et al. Longterm safety evaluation of continuous intraocular delivery of aflibercept by the intravitreal gene therapy candidate ADVM-022 in nonhuman primates. Transl Vis Sci Technol. 2021;10:34.

31. Gelfman CM, Grishanin R, Bender KO, Nguyen A, Greengard J, Sharma P, et al. Comprehensive preclinical assessment of ADVM-022, an intravitreal anti-VEGF gene therapy for the treatment of neovascular AMD and diabetic macular edema. J Ocul Pharm Ther. 2021;37:181-90.

32. Kiss S, Roller CH, Turpcu A, Chung C, Osborne A. ADVM-022 Intravitreal Gene Therapy for Neovascular AMD-Results from the Phase 1 OPTIC Study, Presented at the American Society of Gene and Cellular Therapy (ASGCT) 24th Annual Meeting. 2021

33. Khanani AM, Kiss S, Turpcu A, Hoang C, Osborne A; Phase 1 Study of Intravitreal Gene Therapy ADVM-022 for neovascular AMD (OPTIC Trial). Invest. Ophthalmol. Vis. Sci. 2020;61:1154.

34. Busbee BG, Boyer DS, Khanani AM, et al; Phase 1 Study of Intravitreal Gene Therapy with ADVM-022 for neovascular AMD (OPTIC Trial). Invest. Ophthalmol. Vis. Sci. 2021;62:352.

35. Kiss S, Grishanin R, Nguyen A, Rosario R, Greengard JS, Nieves J, et al. Analysis of aflibercept expression in NHPs following intravitreal administration of ADVM-022, a potential gene therapy for nAMD. Mol Ther Methods Clin Dev. 2020;18:345-53.

36. Biotechnologies A. Adverum Provides Update on ADVM-022 and the INFINITY Trial in Patients with Diabetic Macular Edema. 2021.

37. Siddiqui FA, Aziz AA, Khanani AM. Gene Therapy for Neovascular AMD An update on ongoing clinical trials. Retinal Physician. 2020.

38. REGENXBIO Announces Additional Positive Interim Phase $\mathrm{I} / \mathrm{ll}$ a and Long-Term Follow-Up Data of RGX-314 for the Treatment of Wet AMD. Prnewswire.com. Accessed 1 Apr 2021. http://www.prnewswire.com/news-releases/regenxbioannounces-additional-positive-interim-phase-iiia-and-long-term-follow-up-dataof-rgx-314-for-the-treatment-of-wet-amd-301228344.html.

39. Bahadorani $S$, Singer $M$. Recent advances in the management and understanding of macular degeneration. F1000Res. 2017;6:519.

40. Nozaki M, Raisler BJ, Sakurai E, Sarma JV, Barnum SR, Lambris JD, et al. Drusen complement components $\mathrm{C} 3 \mathrm{a}$ and $\mathrm{C} 5 \mathrm{a}$ promote choroidal neovascularization. Am J Ophthalmol. 2006;142:201. 
41. Dreismann AK, McClements ME, Barnard AR, Orhan E, Hughes JP, Lachmann PJ, et al. Functional expression of complement factor I following AAV-mediated gene delivery in the retina of mice and human cells. Gene Ther. 2021;28:265-76.

42. Waheed NK. FOCUS Interim Results: GT005 Gene Therapy Phase I/II Study for the Treatment of Geographic Atrophy. Presented virtually at Angiogenesis, Exudation, and Degeneration 2021. 2021 Feb 12

43. Tan LX, Toops KA, Lakkaraju A. Protective responses to sublytic complement in the retinal pigment epithelium. Proc Natl Acad Sci USA. 2016;113:8789-94.

44. AAVCAGsCD59 for the Treatment of Wet AMD. Clinicaltrials.gov, Accessed 16 Aug 2021. https://clinicaltrials.gov/ct2/show/NCT03585556.

45. Treatment of Advanced Dry Age Related Macular Degeneration With AAVCAGsCD59. Clinicaltrials.gov. Accessed 20 Aug 2021. https://clinicaltrials.gov/ct2/ show $/$ NCT03144999?term $=$ NCT03144999\&rank $=1$

46. Duker JS. Update on Gene Therapy for Complement Inhibition: 1 Year Results of a Phase 1 Study. Presented at Angiogenesis, Exudation, and Degeneration 2019, Miami, FL. 2019 Feb 9.

47. Peng $Y$, Tang $L$, Zhou $Y$. Subretinal injection: A review on the novel route of therapeutic delivery for vitreoretinal diseases. Ophthalmic Res. 2017;58:217-26.

48. Yiu G, Chung SH, Mollhoff IN, Nguyen UT, Thomasy SM, Yoo J, et al. Suprachoroidal and subretinal injections of AAV using transscleral microneedles for retinal gene delivery in nonhuman primates. Mol Ther Methods Clin Dev. 2020;16:179-91.

49. de Smet MD, Lynch JL, Dejneka NS, Keane M, Khan IJ. A subretinal cell delivery method via suprachoroidal access in minipigs: Safety and surgical outcomes. Investig Ophthalmol Vis Sci. 2018;59:311-20.

50. Moisseiev E, Loewenstein A, Yiu G. The suprachoroidal space: from potential space to a space with potential. Clin Ophthalmol. 2016;10:173-8.

51. Willoughby AS, Vuong VS, Cunefare D, Farsiu S, Noronha G, Danis RP, et al. Choroidal changes after Suprachoroidal Injection of Triamcinolone Acetonide in eyes with macular edema secondary to Retinal Vein Occlusion. Am J Ophthalmol. 2018;186:144-51.

52. Emami-Naeini P, Yiu G. Medical and surgical applications for the suprachoroidal space. Int Ophthalmol Clin. 2019;59:195-207.

53. Yiu G, Pecen P, Sarin N, Chiu SJ, Farsiu S, Mruthyunjaya P, et al. Characterization of the choroid-scleral junction and suprachoroidal layer in healthy individuals on enhanced-depth imaging optical coherence tomography. JAMA Ophthalmol. 2014;132:174-81.

54. Ding K, Shen J, Hafiz Z, Hackett SF, Lima E, Silva R, Khan M, et al. AAV8-vectored suprachoroidal gene transfer produces widespread ocular transgene expression. J Clin Investig. 2019;129:4901-11.

55. Shen J, Kim J, Tzeng SY, Ding K, Hafiz Z, Long D, et al. Suprachoroidal gene transfer with nonviral nanoparticles. Sci Adv. 2020;6. https://doi.org/10.1126/ sciadv.aba1606

56. Chung SH, Mollhoff IN, Mishra A, Sin T-N, Ngo T, Ciulla T, et al. Host immune responses after suprachoroidal delivery of AAV8 in nonhuman primate eyes. Hum Gene Ther. 2021;32:682-93.

57. Mehta N, Robbins DA, Yiu G. Ocular inflammation and treatment emergent adverse events in retinal gene therapy. Int Ophthalmol Clin. 2021;61:151-77.

58. Zaiss A-K, Liu Q, Bowen GP, Wong NCW, Bartlett JS, Muruve DA. Differential activation of innate immune responses by adenovirus and adeno-associated virus vectors. J Virol. 2002;76:4580-90.

59. Vandamme C, Adjali O, Mingozzi F. Unraveling the complex story of immune responses to AAV vectors trial after trial. Hum Gene Ther. 2017;28:1061-74.

60. Khabou H, Cordeau C, Pacot L, Fisson S, Dalkara D. Dosage thresholds and influence of transgene cassette in adeno-associated virus-related toxicity. Hum Gene Ther. 2018;29:1235-41.

61. Mendell JR, Campbell K, Rodino-Klapac L, Sahenk Z, Shilling C, Lewis S, et al. Dystrophin immunity in Duchenne's muscular dystrophy. N. Engl J Med. 2010;363:1429-37.

62. Xiong W, Wu DM, Xue Y, Wang SK, Chung MJ, Rana P, et al. AAV cis-regulatory sequences are correlated with ocular toxicity. Proc Natl Acad Sci USA 2019;116:5785-94.

63. Heier JS, Kherani S, Desai S, Dugel P, Kaushal S, Cheng SH, et al. Intravitreous injection of AAV2-sFLT01 in patients with advanced neovascular age-related macular degeneration: a phase 1, open-label trial. Lancet. 2017;390:50-61.

64. Maguire AM, Russell S, Wellman JA, Chung DC, Yu Z-F, Tillman A, et al. Efficacy, safety, and durability of voretigene neparvovec-rzyl in RPE65 mutationassociated inherited retinal dystrophy: Results of phase 1 and 3 trials. Ophthalmology. 2019;126:1273-85.

65. Xue K, Jolly JK, Barnard AR, Rudenko A, Salvetti AP, Patrício MI, et al. Beneficial effects on vision in patients undergoing retinal gene therapy for choroideremia. Nat Med. 2018;24:1507-12.
66. Seitz IP, Michalakis S, Wilhelm B, Reichel FF, Ochakovski GA, Zrenner E, et al. Superior retinal gene transfer and biodistribution profile of subretinal versus intravitreal delivery of AAV8 in nonhuman primates. Investig Ophthalmol Vis Sci. 2017:58:5792-801.

67. Yiu G. Genome editing in retinal diseases using CRISPR technology. Ophthalmol Retin. 2018;2:1-3.

68. Komor AC, Kim YB, Packer MS, Zuris JA, Liu DR. Programmable editing of a target base in genomic DNA without double-stranded DNA cleavage. Nature. 2016;533:420-4.

69. Gaudelli NM, Komor AC, Rees HA, Packer MS, Badran AH, Bryson DI, et al. Programmable base editing of A.T to G.C in genomic DNA without DNA cleavage. Nature. 2017:551:464-71.

70. Anzalone AV, Randolph PB, Davis JR, Sousa AA, Koblan LW, Levy JM, et al. Searchand-replace genome editing without double-strand breaks or donor DNA. Nature. 2019;576:149-57.

71. Latella MC, Di Salvo MT, Cocchiarella F, Benati D, Grisendi G, Comitato A, et al. In vivo editing of the human mutant Rhodopsin gene by electroporation of Plasmid-based CRISPR/Cas9 in the mouse retina. Mol Ther Nucleic Acids. 2016:5:e389.

72. Tsai $Y-T, W u$ W-H, Lee T-T, Wu W-P, Xu CL, Park KS, et al. Clustered regularly interspaced short palindromic repeats-based genome surgery for the treatment of autosomal dominant retinitis pigmentosa. Ophthalmology. 2018;125:1421-30.

73. Hollander Al, Koenekoop RK, Yzer S, Lopez I, Arends ML, Voesenek KEJ, et al Mutations in the CEP290 (NPHP6) gene are a frequent cause of Leber congenital. Am J Hum Gen. 2006;79:556-61.

74. Ruan G-X, Barry E, Yu D, Lukason M, Cheng SH, Scaria A. CRISPR/Cas9-mediated genome editing as a therapeutic approach for Leber congenital amaurosis 10 . Mol Ther. 2017;25:331-41.

75. Yiu G, Tieu E, Nguyen AT, Wong B, Smit-McBride Z. Genomic disruption of VEGF-A expression in human retinal pigment epithelial cells using CRISPR-Cas9 endonuclease. Investig Ophthalmol Vis Sci. 2016;57:5490-7.

76. Chung SH, Mollhoff IN, Nguyen U, Nguyen A, Stucka N, Tieu E, et al. Factors impacting efficacy of AAV-mediated CRISPR-based genome editing for treatment of choroidal neovascularization. Mol Ther Methods Clin Dev. 2020;17:409-17.

77. Keeler AM, Flotte TR. Recombinant adeno-associated virus gene therapy in light of luxturna (and zolgensma and glybera): Where are we, and how did we get here. Annu Rev Virol. 2019;6:601-21.

\section{AUTHOR CONTRIBUTIONS}

Conception or design of the work: AMK. Acquisition, analysis, or interpretation of data: AMK, CYW, CJD, GY, SK, NKW, PKK. Drafted the work or substantively revised it: AMK, MJT, AAA, CYW, CJD, GY, SK, NKW, PKK.

\section{COMPETING INTERESTS}

AMK is a speaker for Genentech, Allergan, and Novartis, and a consultant for Adverum, Aerpio, Allergan, Chengdu Kanghong, DORC, Genentech, Glaukos, Gyroscope, Gemini Therapeutics, Iveric Bio, Kodiak, Novartis, Opthea, Oxurion, Recens Medical, and Regenxbio and received grant support from Adverum, Alkahest, Allergan, Chengdu Kanghong, Genentech, Gyroscope, Gemini Therapeutics, Kato Pharmaceuticals, Kodiak, NGM Biopharmaceuticals, Novartis, Iveric Bio, Opthea, Oxurion, Recens Medical, Roche, and Regenxbio. CYW is a consultant for Allergan/AbbVie, Novartis, Alcon, Alimera Sciences, Genentech, DORC, Regeneron, Regenxbio. CJD is a consultant for Novartis, Regeneron, Genentech, Roche, IvericBio, DORC. GY is a consultant for Alimera, Allergan, Carl Zeiss Meditec, Genentech, Gyroscope Therapeutics, Intergalactic Therapeutics, Iridex, NGM Biopharmaceuticals, Regeneron, Topcon, Verily. SK is a consultant for Adverum, Alcon, Gyroscope, Optos, Regeneron, Genentech/Roche, Regenxbio. NKW is Chief Medical Officer of Gyroscope Therapeutics. PKK is a consultant for Allergan/AbbVie, Bayer, Chengdu Kanghong, Gyroscope, Gemini Therapeutics, Iveric Bio, Kodiak, Novartis, Opthea, Oxurion, Regeneron, and Regenxbio. MJT and AAA declare no potential competing interests.

\section{ADDITIONAL INFORMATION}

Correspondence and requests for materials should be addressed to Arshad $M$ Khanani.

Reprints and permission information is available at http://www.nature.com/ reprints

Publisher's note Springer Nature remains neutral with regard to jurisdictional claims in published maps and institutional affiliations. 